\title{
Summary of the NACI Update on the
}

recommended use of Human Papillomavirus (HPV) vaccine: Nine-valent HPV vaccine twodose immunization schedule and the use of HPV vaccines in immunocompromised populations

\author{
SL Deeks ${ }^{1,2}, \mathrm{MC}$ Tunis $^{3}$, S Ismail ${ }^{3}$ on behalf of the National Advisory Committee on Immunization \\ (NACl)*
}

\begin{abstract}
Background: Human papillomavirus (HPV) infections are the most common sexually transmitted infections. In the absence of vaccination, it is estimated that $75 \%$ of sexually active Canadians will have an HPV infection at some point in their lives. HPV vaccine programs were first recommended by Canada's National Advisory Committee on Immunization (NACI) in 2007. In addition to the existing HPV vaccine options in Canada, NACI recently recommended the use of a newly authorized nine-valent HPV (HPV9) vaccine according to a 3-dose immunization schedule for the prevention of HPV types 6-, 11-, 16-, 18-, 31-, 33-, 45-, 52- and 58-related cancers and anogenital warts in females aged 9 to 45 years and males aged 9 to 26 years. New data have emerged evaluating a 2-dose immunization schedule for HPV9 vaccine in males and females, which $\mathrm{NACl}$ reviewed in order to provide timely guidance on the possibility of a 2-dose immunization schedule for HPV9 vaccine. Recently, a growing number of studies have also specifically explored the responses of immunocompromised subgroups to HPV vaccines, which also triggered a NACl literature review and updated recommendations on this topic.
\end{abstract}

Objectives: To review evidence for a 2-dose immunization schedule of the HPV9 vaccine and provide recommendations on vaccine schedule; and to summarize evidence from a recent $\mathrm{NACl}$ literature review on the use of HPV vaccines in immunocompromised populations and provide recommendations for HPV vaccine use in these groups.

Methods: The NACI HPV Working Group reviewed results from a clinical trial of HPV9 vaccine administered with a 2-dose immunization schedule in males and females (protocol V503-010) and performed a literature review on the topic of HPV immunization of immunocompromised populations. The $\mathrm{NACl}$ literature review and the $\mathrm{NACl}$ statement were published separately.

Results: Only one study investigated a 2-dose immunization schedule with HPV9 vaccine, a large manufacturer-sponsored randomized controlled trial (protocol V503-010) of good quality. Taken in context of studies with other HPV vaccines, NACl considered this study to be a sufficient evidence base for recommendations. Through a comprehensive literature review, 27 studies were identified for evidence synthesis including reports on vaccine immunogenicity, safety, or both for immunocompromised populations.

Conclusions: Based on the evidence reviewed, $\mathrm{NACl}$ issued new recommendations for the use of HPV9 vaccine with a 2-dose immunization schedule at 0, 6-12 months in young females and males and updated the grade of evidence for the use of HPV vaccines in immunocompromised populations.

\begin{abstract}
Affiliations
${ }^{1} \mathrm{NACl}$ HPV Working Group Chair, Toronto, ON

${ }^{2}$ Immunization and Vaccine Preventable Diseases, Public Health Ontario, Toronto, ON

${ }^{3}$ Centre for Immunization and Respiratory Infectious Diseases, Public Health Agency of Canada, Ottawa, ON
\end{abstract}

*Correspondence: naci-ccni@ phac-aspc.gc.ca
Suggested citation: Tunis MC, Deeks SL, Ismail S on behalf of the National Advisory Committee on Immunization (NACl). Summary of the NACI Update on the recommended use of Human Papillomavirus (HPV) vaccine: Nine-valent HPV vaccine two-dose immunization schedule and the use of HPV vaccines in immunocompromised populations. Can Commun Dis Rep. 2017;43(6):138-42. https://doi.org/10.14745/ccdr.v43i06a04 


\section{Introduction}

HPV infections are the most common sexually transmitted infections. In the absence of vaccination, it is estimated that $75 \%$ of sexually active Canadians will have a human papillomavirus (HPV) infection at some point in their lives. Quadrivalent (HPV4) and bivalent (HPV2) HPV vaccines have been authorized for use in Canada since 2006 and 2010, respectively. The National Advisory Committee on Immunization (NACl) has previously recommended HPV4 vaccination in males and females according to a 3-dose (0, 2, 6 months) or a 2-dose $(0,6$ months) immunization schedule, or HPV2 vaccination for females according to a 3-dose $(0,1,6$ months) or a 2-dose $(0$, 6 months) immunization schedule, depending on the age and health status of the recipient. In February 2015, a nine-valent HPV vaccine (HPV9) (Gardasil ${ }^{\circledR 9}$, Merck Canada, Inc.) was authorized for use in Canada for the prevention of HPV types 6-, 11-, 16-, 18-, 31-, 33-, 45-, 52- and 58-related cancers and anogential warts in females aged 9 to 45 years and males aged 9 to 26 years; this vaccine was recommended by $\mathrm{NACl}$ as a 3-dose immunization schedule in a NACI Statement (1) and Canada Communicable Disease Report (CCDR) summary (2) published in July 2016. Immunization against HPV types 16 and 18 can prevent approximately $70 \%$ of anogenital cancers and $60 \%$ of high-risk precancerous cervical lesions. It is estimated that immunization against the additional five HPV types contained in the HPV9 vaccine can prevent up to an additional $14 \%$ of anogenital cancers and up to $30 \%$ of high-risk precancerous cervical lesions.

$\mathrm{NACl}$ reviewed new clinical trial evidence for a 2-dose immunization schedule of HPV9 vaccine in order to provide timely guidance on the possibility of a 2-dose immunization schedule for HPV9 vaccine. Previous NACI Statements have also discussed the use of HPV vaccines in immunocompromised populations, but this has been largely in the absence of strong evidence for these groups. Recently, a growing number of studies have specifically explored the responses of immunocompromised subgroups to HPV vaccines, which triggered a NACl Literature Review on this topic and advice in the statement. This paper summarizes the recent $\mathrm{NACl}$ Statement on the evidence and recommendations for a 2-dose immunization schedule for HPV9 vaccine (3) and the $\mathrm{NACl}$ Literature Review that updates the evidence on the use of HPV vaccines in immunocompromised persons (4). These new recommendations are published in the full NACI Statement (3) and also in the HPV chapter of the Canadian Immunization Guide, where all current HPV recommendations from $\mathrm{NACl}$ are synthesized and presented together (5).

\section{Methods}

$\mathrm{NACl}$ reviewed key questions on HPV9 vaccine as proposed by the HPV Working Group, including: the safety, immunogenicity and efficacy of the HPV9 vaccine with a 2-dose immunization schedule. Only one manufacturer-sponsored clinical trial was identified (protocol V503-010) (6). The NACI HPV Working Group reviewed detailed study methods and results obtained from the vaccine manufacturer. Two independent reviewers appraised the study for internal validity to assign the level and quality of evidence, which was validated by the Working Group Chair and subjected to a modified peer review process by the Working Group and $\mathrm{NACl}$.

$\mathrm{NACl}$ also reviewed key questions for a literature review on HPV vaccines in immunocompromised populations as proposed by the HPV Working Group, including the specific question: "Do immunization outcomes differ for subgroups of immunocompromised populations?" A literature review was conducted according to established $\mathrm{NACl}$ methodology (7). Details of this literature review and associated methodology (search terms, inclusion/exclusion criteria, databases, number of results) can be found in the $\mathrm{NACl}$ Literature Review for HPV Immunization of Immunocompromised Populations (4). Proposed recommendations were developed based on the literature review. $\mathrm{NACl}$ approved specific evidence-based recommendations and elucidated the rationale and relevant considerations in the statement update.

\section{Results}

A large randomized controlled trial of good quality, demonstrating that immunogenicity following a 2-dose immunization schedule among females and males 9 to 14 years of age, was not inferior to a 3-dose schedule in women 16 to 26 years of age. Taken in context of studies on 2-dose immunization schedules with other HPV vaccines, NACl considered the body of evidence to be sufficient to issue recommendations on a 2-dose immunization schedule for HPV9 vaccine.

On the topic of immunocompromised populations, the literature review identified 27 studies for evidence synthesis including reports on immunogenicity, safety, or both (4). Studies were grouped broadly into two categories: HIV infected populations and groups receiving therapeutic immunosuppression. Cohort study was the predominant experimental design and most included a low participant number with quality ranging from good to poor.

Immunosuppressive regimes were typically reported for patients with acquired therapeutic immunodeficiency, but unfortunately these studies did not often stratify results according to treatment modality. The extent of effective immunosuppression was not measured or reported in any study, but CD4 counts were typically provided for studies of HIV infected patients. All studies used HPV2 or HPV4 vaccines with a routine 3-dose schedule, except one study which provided an additional fourth dose. No study used the recently-authorized HPV9 vaccine.

\section{Conclusion}

Based on the evidence reviewed, $\mathrm{NACl}$ issued three recommendations on the use of a 2-dose immunization schedule in immunocompetent populations and the use of HPV vaccines in immunocompromised populations (Text box). 


\section{RECOMMENDATIONS FOR 2-DOSE HPV9 SCHEDULE IN IMMUNOCOMPETENT POPULATIONS}

Recommendation 1: Immunocompetent Females and Males 9-14 Years of Age

$\mathrm{NACl}$ recommends that HPV9 vaccine should be offered according to either a 2-dose or 3-dose immunization schedule in immunocompetent females and males 9 to 14 years of age (as with HPV2 or HPV4 vaccines in females, and HPV4 vaccine in males in this population) - NACI Evidence Grade B Recommendation (fair evidence to recommend immunization).

$\mathrm{NACl}$ concludes that there is now fair evidence to recommend a 2-dose immunization schedule with HPV9 vaccine, although evidence is limited in quantity. Therefore, based on the current evidence reviewed for this and previous Advisory Committee Statements, $\mathrm{NACl}$ concludes that there is fair evidence to recommend either a 2-dose or a 3-dose immunization schedule with HPV9 vaccine (Evidence Grade B) and that there is good evidence to recommend either a 2-dose or a 3-dose immunization schedule with HPV2 or HPV4 vaccines (in females) and HPV4 vaccine (in males). In a 2-dose HPV immunization schedule with any HPV vaccine authorized for use in Canada, the second dose should be administered at least 24 weeks (6 months) after the first dose. Although long-term follow-up data are not currently available for a 2-dose schedule with HPV9, a study is currently underway to evaluate the duration of vaccine responses up to 36 months. As further evidence becomes available, the grade of this recommendation may change. There is no evidence to suggest that individuals will respond differently to HPV9 vaccine compared to either HPV2 or HPV4 vaccines.

Recommendation 2: Immunocompetent Females and Males $\geq 15$ Years of Age

$\mathrm{NACl}$ continues to recommend that HPV9 vaccine should be offered according to a 3-dose immunization schedule in immunocompetent females and males 15 years of age and older (as with HPV2 or HPV4 vaccines in females and HPV4 vaccine in males) - NACI Evidence Grade B Recommendation (fair evidence to recommend immunization).

There are currently no studies directly evaluating a 2-dose immunization schedule for HPV9 vaccine in males and females 15 years of age and older. Therefore, a 3-dose schedule continues to be recommended in these populations. This recommendation is outlined in the previous statement Updated Recommendations on Human Papillomavirus (HPV) Vaccines: 9-valent HPV vaccine and clarification of minimum intervals between doses in the HPV immunization schedule. However, a recent study in India has suggested that 2 doses of HPV4 vaccine may be immunogenic in females aged 10-18 years, and NACl will continue to review similar evidence as it emerges in order to identify the optimal HPV9 immunization schedule for persons 15 years of age and older.

\section{RECOMMENDATIONS FOR HPV VACCINATION IN PERSONS WHO ARE IMMUNOCOMPROMISED AS A RESULT OF DISEASE OR MEDICATIONS}

Recommendation 3:

$\mathrm{NACl}$ continues to recommend that HPV vaccines be administered using a 3-dose schedule in immunocompromised populations according to existing age guidelines - NACl Evidence Grade B Recommendation for HPV2 and HPV4 vaccine (fair evidence to recommend immunization); NACI Evidence Grade I Recommendation for HPV9 vaccine (insufficient evidence in either quantity and/or quality to make a recommendation, however other factors may influence decision-making).

While NACl's recommendation regarding HPV2 and HPV4 vaccines in this population remains unchanged, based on the Literature Review conducted to inform these recommendations the Evidence Grade on which the recommendation is based has been upgraded from a Grade I (insufficient evidence in either quantity and/or quality to make a recommendation, however other factors may influence decision-making) to a Grade B (fair evidence to make a recommendation). Evidence does not suggest there are any unique safety concerns in using HPV2 or HPV4 vaccines for immunocompromised populations. There are currently no studies directly evaluating the immunogenicity, efficacy, or safety of HPV9 vaccine in immunocompromised populations with either a 3-dose or a 2-dose schedule (therefore the Evidence Grade on which the recommendation is based is Grade I). However, there is no evidence to suggest that individuals would respond differently to HPV9 vaccine compared to either HPV2 or HPV4 vaccines. As further evidence becomes available, the grade of this recommendation may change.

There are currently no published studies exploring a 2-dose HPV immunization schedule in immunocompromised populations. $\mathrm{NACl}$ concludes that there is fair evidence demonstrating that the immunogenicity of HPV2 and HPV4 vaccines can be diminished in immunocompromised populations following a 3-dose immunization schedule, although the antibody responses still typically exceed those resulting from natural infection in immunocompetent individuals. Therefore, although the immunogenicity and efficacy have not been fully characterised in all immunocompromised populations, individuals who are immunocompromised are expected to derive benefit from these vaccines and $\mathrm{NACl}$ continues to recommend vaccination of these groups using a 3-dose schedule to provide protection. 
Table 1 summarizes the above recommendations according to when to give HPV vaccine and to whom. HPV immunization may be completed with HPV2, HPV4 or HPV9 vaccines in females and HPV4 or HPV9 vaccines in males, according to the immunization schedules summarized in this table. Where possible, the same vaccine should be used to complete the series. If completion of the series with the same vaccine is not possible, the HPV2, HPV4 or HPV9 vaccine may be used to complete the series in females and the HPV4 or HPV9 vaccine may be used to complete the series in males. The HPV9 vaccine among immunocompetent 9 to 26 year olds is expected to provide similar protective efficacy against genotypes contained in the HPV4 vaccine. Moreover, HPV9 vaccine protects against the additional five HPV types not contained in HPV4 vaccine (HPV 31, 33, 45, 52 and 58).

Table 1: NACI Recommendations for the HPV Immunization Schedule

\begin{tabular}{|c|c|c|}
\hline $\begin{array}{l}\text { Recommended } \\
\text { groups }\end{array}$ & $\begin{array}{l}\text { Recommended } \\
\text { immunization } \\
\text { schedule }\end{array}$ & $\begin{array}{c}\text { HPV vaccines and } \\
\text { NACI Evidence } \\
\text { Grade (7) }\end{array}$ \\
\hline $\begin{array}{l}\text { Healthy } \\
\text { (immunocompetent, } \\
\text { non-HIV infected) } \\
\text { females } 9-14 \text { years } \\
\text { of age (and healthy } \\
\text { females } \geq 15 \text { years of } \\
\text { age in whom the first } \\
\text { dose was administered } \\
\text { between } 9-14 \text { years } \\
\text { of age) }\end{array}$ & $\begin{array}{l}\text { 2- or 3-dose } \\
\text { schedule }\end{array}$ & $\begin{array}{l}\text { HPV2 or HPV4 } \\
\text { (Grade A) } \\
\text { HPV9 (Grade B) }\end{array}$ \\
\hline $\begin{array}{l}\text { Healthy } \\
\text { (immunocompetent, } \\
\text { non-HIV infected) } \\
\text { females } \geq 15 \text { years of } \\
\text { age }\end{array}$ & 3-dose schedule & $\begin{array}{l}\text { HPV2 or HPV4 } \\
\text { (Grade A) or } \\
\text { HPV9 (Grade B) }\end{array}$ \\
\hline $\begin{array}{l}\text { Healthy } \\
\text { (immunocompetent, } \\
\text { non-HIV infected) } \\
\text { males } 9-14 \text { years } \\
\text { of age (and healthy } \\
\text { males } \geq 15 \text { years of } \\
\text { age in whom the first } \\
\text { dose was administered } \\
\text { between } 9-14 \text { years } \\
\text { of age) }\end{array}$ & $\begin{array}{l}\text { 2- or 3-dose } \\
\text { schedule }\end{array}$ & $\begin{array}{l}\text { HPV4 or } \\
\text { HPV9 (Grade B) }\end{array}$ \\
\hline $\begin{array}{l}\text { Healthy } \\
\text { (immunocompetent, } \\
\text { non-HIV infected) } \\
\text { males } \geq 15 \text { years } \\
\text { of age }\end{array}$ & 3-dose schedule & $\begin{array}{l}\text { HPV4 or } \\
\text { HPV9 (Grade B) }\end{array}$ \\
\hline $\begin{array}{l}\text { Immunocompromised } \\
\text { individuals and } \\
\text { immunocompetent } \\
\text { HIV infected } \\
\text { individuals }\end{array}$ & 3-dose schedule & $\begin{array}{l}\text { HPV2 or HPV4 in } \\
\text { females (Grade B); } \\
\text { HPV4 in males } \\
\text { (Grade B); } \\
\text { HPV9 in females or } \\
\text { males (Grade I) }\end{array}$ \\
\hline
\end{tabular}

Efforts should be made to administer HPV vaccines at the recommended intervals. When an abbreviated schedule is required, minimum intervals between vaccine doses should be met. In a 3-dose schedule, the minimum interval between the first and second doses of vaccine is four weeks, the minimum interval between the second and third doses of vaccine is 12 weeks and the minimum interval between the first and last doses in either a 2 -dose or 3 -dose schedule is 24 weeks.

\section{Authors' statement}

The NACl Statement summarized in this article was prepared by Dr. M. Tunis, Dr. S. Ismail, Dr. S. Deeks and approved by NACI.

\section{Conflict of interest}

None.

\section{Acknowledgements}

HPV Working Group Members: Dr. S. Deeks (Chair), Dr. E. Castillo, Dr. S. Dobson, Dr. M. Krajden, Dr. G. Ogilvie and Dr. C. Sauvageau

NACI Members: Dr. I. Gemmill (Chair), Dr. C. Quach (Vice-Chair), Dr. N. Dayneka, Dr. S. Deeks, Dr. B. Henry, Ms. S. Marchant-Short, Dr. M. Salvadori, Dr. N. Sicard, Dr. W. Vaudry, Dr. D. Vinh and Dr. R. Warrington

Liaison Representatives: Dr. J. Blake (Society of Obstetricians and Gynaecologists of Canada), Dr. J. Brophy (Canadian Association for Immunization Research and Evaluation [CAIRE]), Dr. A. Cohn (Centers for Disease Control and Prevention, United States), Ms. T. Cole (Canadian Immunization Committee), Dr. J. Emili (College of Family Physicians of Canada), Dr. M. Lavoie (Council of Chief Medical Officers of Health), Dr. C. Mah (Canadian Public Health Association), Dr. D. Moore (Canadian Paediatric Society), Dr. A. Pham-Huy (Association of Medical Microbiology and Infectious Disease [AMMI] Canada) and Ms. T. Cole (Canadian Immunization Committee)

Former Liaison Representatives: Ms. E. Sartison (Canadian Immunization Committee)

Ex-Officio Representatives: Dr. (LCdr) K. Barnes (National Defence and the Canadian Armed Forces), Ms. G. Charos (Centre for Immunization and Respiratory Infectious Diseases [CIRID], Public Health Agency of Canada [PHAC]), Dr. G. Coleman (Biologics and Genetic Therapies Directorate [BGTD], Health Canada [HC]), Dr. J. Gallivan (Marketed Health Products Directorate [MHPD], HC), Ms. J. Pennock (CIRID, PHAC), Dr. T. Wong (First Nations and Inuit Health Branch [FNIHB], HC)

$\mathrm{NACl}$ gratefully acknowledges the contribution of Ms. C. Jensen (CIRID, PHAC), Mr. J. Nkanza (CIRID, PHAC) and Ms. C. Smalley (Health Library, HC). 


\section{Funding}

The work of National Advisory Committee on Immunization is supported by the Public Health Agency of Canada.

\section{References}

1. Government of Canada [Internet]. Updated Recommendations on Human Papillomavirus (HPV) Vaccines: 9-valent HPV vaccine and clarification of minimum intervals between doses in the HPV immunization schedule. Ottawa: NACl; 2016 [updated 2016 Jul 28; cited 2017 Mar 21]. Available from: http://www.healthycanadians.gc.ca/ publications/healthy-living-vie-saine/human-papillomavirus9-valent-vaccine-update-recommendation-mises-a-jourrecommandations-papillome-humain-vaccin-nonavalent/ index-eng.php.

2. Tunis MC, Deeks SL. on behalf of the National Advisory Committee on Immunization. Summary of the National Advisory Committee on Immunization's Updated Recommendations on Human Papillomavirus (HPV) vaccines: Nine-valent HPV vaccine and clarification of minimum intervals between doses in the HPV immunization schedule. Can Commun Dis Rep. 2016;42(7):14951. Available from: http://www.phac-aspc.gc.ca/publicat/ccdrrmtc/16vol42/dr-rm42-7/ar-03-eng.php.

3. Government of Canada [Internet]. Updated Recommendations on Human Papillomavirus (HPV) Vaccines: 9-valent HPV vaccine 2-dose immunization schedule and the use of HPV vaccines in immunocompromised populations. Ottawa: NACl; 2017 [update 2017 May 29] Available from: https://www.canada.ca/en/ public-health/services/publications/healthy-living/updatedrecommendations-human-papillomavirus-immunizationschedule-immunocompromised-populations.html.

4. Government of Canada [Internet]. NACI Literature Review for HPV Immunization of Immunocompromised Populations. Ottawa: $\mathrm{NACl} ; 2017$ [updated 2017 May 29] Available from: https:// www.canada.ca/en/public-health/services/publications/ healthy-living/literature-review-human-papillomavirusimmunization-immunocompromised-populations.html.

5. National Advisory Committee on Immunization. Canadian Immunization Guide: Part 4: Human papillomavirus vaccine. Ottawa (ON): Public Health Agency of Canada [updated 2017 May 29]. https://www.canada.ca/fr/sante-publique/services/ publications/vie-saine/guide-canadien-immunisation-partie4-agents-immunisation-active/page-9-vaccin-contre-viruspapillome-humain.html.

6. ClinicalTrials.gov [Internet]. Merck Sharp \& Dohme Corp. A Phase III Study of a 2-dose Regimen of a Multivalent Human Papillomavirus (HPV) Vaccine (V503), Administered to 9 to 14 Year-olds and Compared to Young Women, 10 to 26 Years Old (V503-101). [updated 2017 Mar 9; cited 2017 Mar 21]. Available from: https://clinicaltrials.gov/ct2/show/study/NCT01984697.

7. Public Health Agency of Canada [Internet]. Evidence-based recommendations for immunization: Methods of the National Advisory Committee on Immunization. Ottawa: NACl; 2009 [updated 2009 Feb 2; cited 2017 Mar 21]. Available from: http:// www.phac-aspc.gc.ca/publicat/ccdr-rmtc/09vol35/acs-1/indexeng.php.

\section{Two publications of the \\ Canadian Guidelines on Sexually Transmitted Infections (CGSTI)}

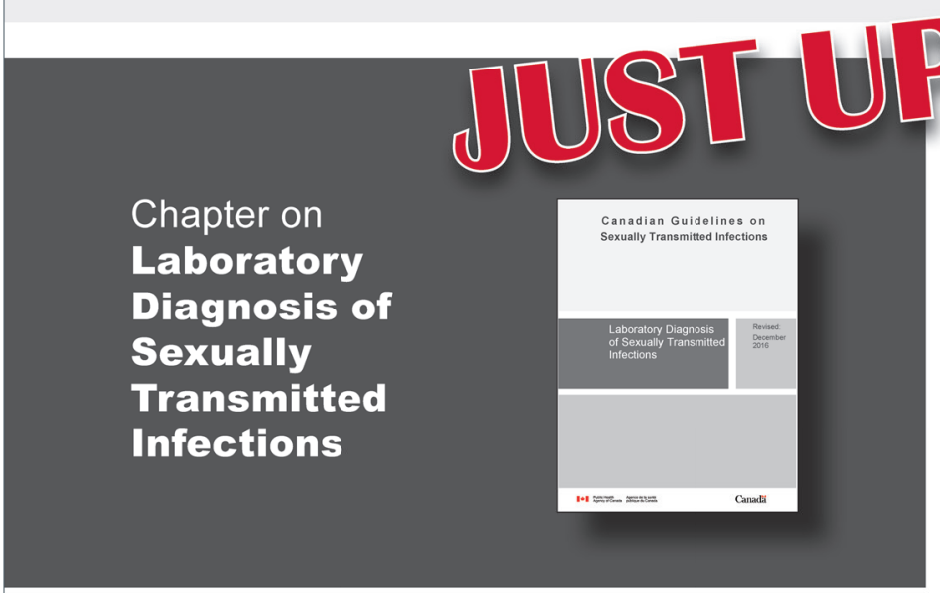
PDATED!

Chapter on

Laboratory

Diagnosis of

Sexually

Transmitted

Infections
2016 Updates Summary for key content changes to the CGSTI

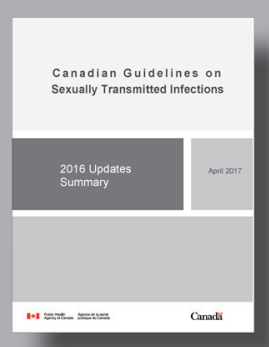

Ensure you are using the most current recommendations Publications are FREE. Visit Canada.ca and search "CGSTI" 